\title{
Computer-aided diagnosis in mammography: classification of mass and normal tissue by texture analysis
}

\author{
Arthur Petrosian, Heang-Ping Chan, Mark A Helvie, Mitchell M Goodsitt \\ and Dorit D Adler \\ Department of Radiology, University of Michigan, Ann Arbor, MI, USA
}

Received 24 January 1994, in final form 16 August 1994

\begin{abstract}
Computer-aided diagnosis schemes are being developed to assist radiologists in mammographic interpretation. In this study, we investigated whether texture features could be used to distinguish between mass and non-mass regions in clinical mammograms. Forty-five regions of interest (ROIS) containing true masses with various degrees of visibility and 135 ROIS containing normal breast parenchyma were extracted manually from digitized mammograms as case samples. Spatial-grey-level-dependence (SGLD) matrices of each RoI were calculated and eight texture features were calculated from the SGLD matrices. The correlation and class-distance properties of extracted texture features were analysed.

Selected texture features were input into a modified decision-tree classification scheme. The performance of the classifier was evaluated for different feature combinations and orders of features on the tree. A classification accuracy of about $89 \%$ sensitivity and $76 \%$ specificity was obtained for ordered features, sum average, correlation, and energy, during the training procedure. With a leave-one-out method, the test result was about $76 \%$ sensitivity and $64 \%$ specificity. The results of this preliminary study demonstrate the feasibility of using texture information for classification of mass and normal breast tissue, which will be likely to be useful for classifying true and false detections in computer-aided diagnosis programmes.
\end{abstract}

\section{Introduction}

It is known that images of many target objects or lesions are characterized by unique textural and shape properties. Computerized pattern-recognition techniques have been applied to mammographic images (Hand et al 1979, Magnin et al 1986, Chan et al 1987, Fam et al 1988, Lai et al 1989, Caldwell et al 1990, Davies et al 1990, Yin et al 1991, Cheng et al 1993) as well as other types of medical image (Kruger et al 1974, Garra et al 1989, Cheng et al 1991, Goldberg et al 1992). Detection of mammographic abnormalities using morphological features has been reported (Hand et al 1979, Chan et al 1989, Lai et al 1989, Davies et al 1990, Brzakovic et al 1990, Yin et al 1991, Mascio et al 1993). Investigators have also explored the extraction of image statistical and textural information and classification of normal and disease patterns with discriminant analysis or neuralnetwork techniques (Kruger et al 1974, Magnin et al 1986, Garra et al 1989, Katsuragawa et al 1988, Caldwell et al 1990, Cheng et al 1991, Goldberg et al 1992, Dhawan et al 1993, Cheng et al 1993). More recently, it was reported that edge-gradient orientation in combination with Laws texture features could be used to effectively detect spiculated masses on mammograms (Kegelmeyer et al 1994). The methods utilizing texture features take advantage of the fact that computers are better than human observers in analysing second-order statistical features. 
The purpose of the present study was to extract texture features from regions of interest (ROIS) on mammograms and use those features to determine whether the ROI contained a mass or normal tissue. The features were derived from the spatial-grey-leveldependence (SGLD) matrix (Haralick et al 1973, Pratt 1978, Conners 1979), also known as the concurrence or co-occurrence matrix. The classification capabilities of the features were analysed by their correlation coefficients and class distances. Groups of three features were selected and put into a decision-tree classification scheme (Jain 1989, Agin et al 1975). The decision-tree classification approach is based on a so-called distribution-free method. That is, it does not require knowledge of any a priori probability distribution functions. The advantages of this method include its simplicity, requiring relatively low complexity of calculations, as well as the fact that, unlike many other algorithms that require a training stage, it is guaranteed to converge whether or not the feature space is linearly separable. Making use of a modified decision-tree classification algorithm which is described step by step below in section 2 , we considered training sets containing case samples of four different patterns, mass, dense fibroglandular, mixed glandular and fatty, and fatty breast parenchyma, and classified them into normal and abnormal categories. The decision tree was optimized by varying the thresholds for the extracted features as well as entering the features into the tree in different orders. Thus, unlike traditional methods (Jain 1989), our method allowed evaluation of the performance of different texture features in different branches separately. It revealed reasons for inefficiencies of specific features and permitted investigation of ways to improve those individual branches either by employing preprocessing procedures or by considering additional features.

\section{Materials and methods}

Mammograms used in this study were selected from patient files in the Department of Radiology at the University of Michigan Hospitals. All mammograms were obtained with Kodak MinR/MRE screen/film systems using dedicated mammographic units with molybdenum anodes and $0.03 \mathrm{~mm}$ thick molybdenum filters. The mammograms were digitized with a Lumisys laser film scanner with a pixel size of $0.1 \mathrm{~mm} \times 0.1 \mathrm{~mm}$ and 4096 grey levels. The digitizer was calibrated so that the pixel values were linearly proportional to the optical density in the range of $0.1-2.8$ optical-density units (OD) at $0.001 \mathrm{oD} /$ pixel value. The gradient of the calibration curve falls off gradually outside this optical density range. In this study, 45 clinical mammograms were selected from our database. Each of the selected mammograms contained a mass proven to be malignant by biopsy. No criterion was imposed on the morphology of the masses so that the selected cases included both spiculated $(n=27)$ and non-spiculated $(n=18)$ masses.

Four different ROIs, each of $256 \times 256$ pixels, were manually extracted from each mammogram. One of the RoIs contained the true mass, hereafter referred to as $M$. The other three RoIs contained normal breast parenchyma. One, denoted N1, contained a small dense region resulting, for example, from imaging crossing vessels, vessels on end, or overlapping tissue. A second, denoted $\mathrm{N} 2$, contained dense fibroglandular tissue or overlapping breast structures that might simulate a mass. The third, denoted $\mathrm{N} 3$, contained fatty tissue. An example of each of these ROIs is shown in figure 1 . We also considered an $\mathrm{N}$ class which included 45 ROIs, with 15 Rols from each of the N1, N2, and $\mathrm{N} 3$ groups. The 15 RoIs from each of the $\mathrm{N}$ groups were randomly selected from each group with the constraint that no two N-type ROIs were obtained from the same mammogram.

All Rors were ranked, on a scale of one to 10 (one, least likely; 10 , most likely), by two experienced radiologists (MAH, DDA) regarding the likelihood of containing a mass. In the 


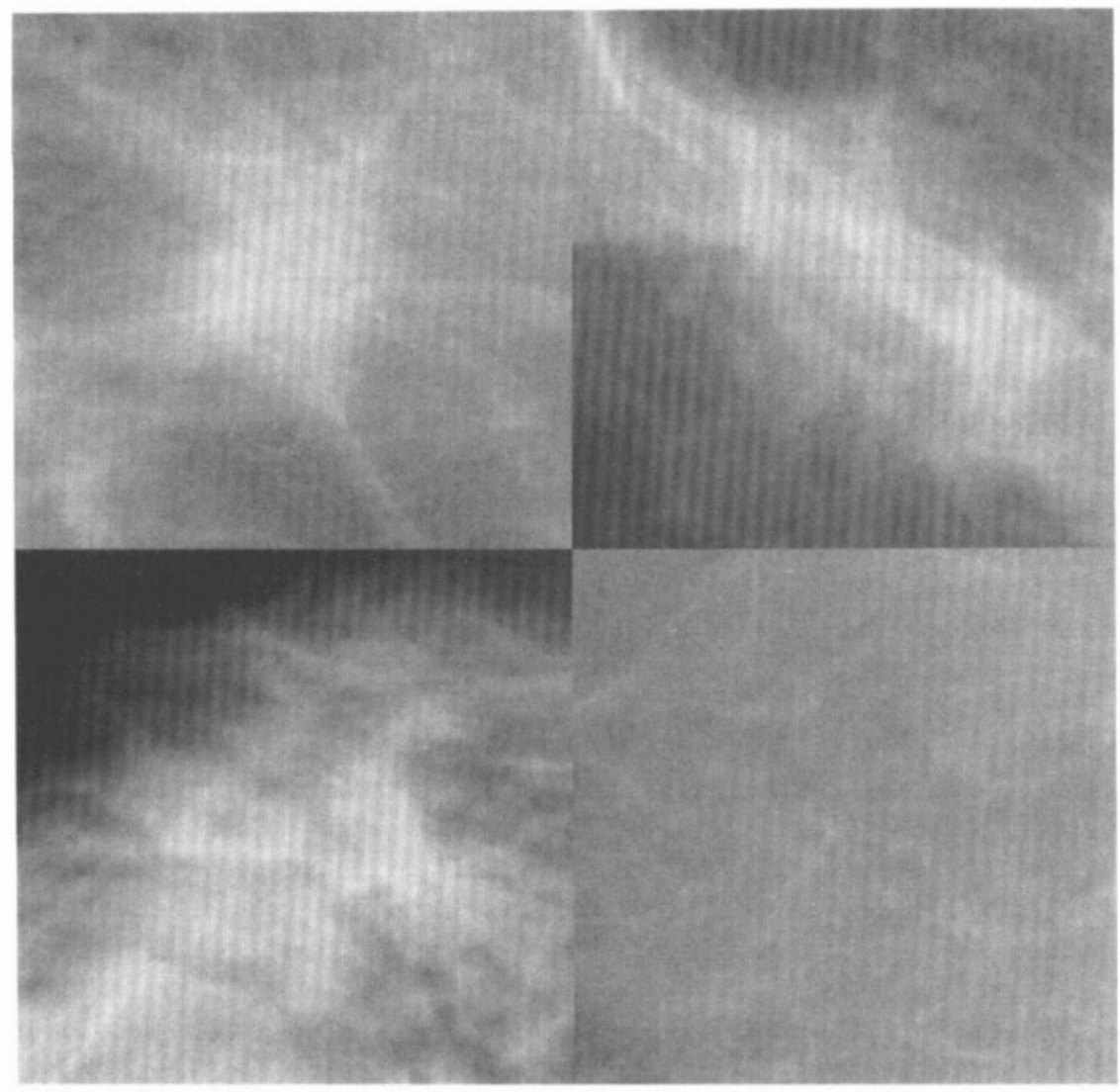

Figure 1. An example of the mass and non-mass regions manually selected from one of the clinical mammograms used in this study. The four Rols are upper left, mass ( $M$ ); upper right, normal mixed dense and fatty tissue (N1); lower left, dense fibroglandular tissue (N2); lower right, fatty tissue (N3).

ranking experiment, the radiologists read a single-view mammogram on which the locations of the four RoIs were marked. If the likelihood was greater than one, the radiologists also ranked the visibility of the 'mass' (one, most obvious, 10 , most subtle mass seen on clinical mammograms), and the diameter of the 'mass' was measured. The average ranks and sizes for the four types of ROI are summarized in table 1. The likelihood of containing a mass for the $45 \mathrm{M}$-class RoIs ranged from four to 10 , with a mean of 7.5 . The visibility ranks of the 45 true masses ranged from two to eight with a mean of 4.2 . The diameter of the 45 true masses ranged from $7 \mathrm{~mm}$ to $19 \mathrm{~mm}$ with a mean of $11 \mathrm{~mm}$. Although the ranks are quite subjective and will be observer dependent, they are included here in an attempt to describe the appearance of the masses used in our study relative to those seen on mammograms of our patient population. It should be noted that the radiologists could use the mammographic information surrounding an Rol to determine whether a mass was present whereas the computer was presented with the ROI without the surroundings. About one-third of the $\mathrm{N} 1$ and $\mathrm{N} 2$ Rols were ranked by the radiologists as regions with some 
Table 1. The average ranks of the 45 mass (M) and non-mass (N1, N2, N3) regions. The rank of the likelihood of containing a mass was obtained by averaging the ranks of all 45 Rors for each type. The visibility rank and the size were obtained by averaging the regions with a likelihood rank greater than unity.

\begin{tabular}{lrrrr}
\hline & $\mathrm{M}$ & $\mathrm{N} 1$ & $\mathrm{~N} 2$ & $\mathrm{~N} 3$ \\
\hline Likelihood of containing a mass & 7.5 & 1.6 & 1.6 & $\mathrm{I}$ \\
Visibility & 4.2 & 6.1 & 6.7 & - \\
Size (mm) & 11.1 & 8.6 & 11.3 & - \\
\hline
\end{tabular}

\section{likelihood of containing a mass.}

The two-dimensional SGLD matrix was determined for each ROr image. An SGLD matrix element, $p_{\theta, d}(i, j)$, is the frequency of occurrence or joint probability of the grey-level pairs $i$ and $j$ in a given direction $\theta$ and separated by a distance of $d$ pixels. In this feasibility study, the distance was chosen to be one pixel and the grey levels were grouped into $128 \times 128$ bins (seven bits) in the SGLD matrix. The texture features in four directions $\left(\theta=0^{\circ}, 45^{\circ}, 90^{\circ}\right.$, $\left.135^{\circ}\right)$ were calculated and their average was used as input to the classifier. This choice was made because the dominant features in an ROr with a mass were approximately rotationally invariant.

The eight features evaluated in this study included angular second moment (energy), entropy, correlation, inertia, sum average, surn entropy, difference entropy, and inverse difference moment (local homogeneity) (appendix A). These eight quantities characterize the energy spread about the diagonal of the SGLD matrix. The correlation coefficient between every two features within a class as well as the variance-normalized distance between every two class means was calculated (appendix B) to evaluate the ability of the features to distinguish between two classes.

For classification of the features, we chose to use a three-layer, seven-node decision-tree classifier. The decision-tree classifier as found in the literature (Jain 1989) was based on the normalized average prototype feature matrix. In this study, we proposed a modified optimization algorithm in which the thresholds to be evaluated in each layer were selected from the values of the corresponding feature in the training set. A flow diagram of the modified decision tree using three features for classification is shown in figure 2 . The algorithm for optimization of the decision tree can be summarized as follows.

Step 1. The order of the three selected features is chosen and the features are assigned to the tree nodes.

Step 2. A threshold $\alpha$ is selected for the feature $F_{1}$ in the first layer. $\alpha$ is chosen to be one of the values of $F_{1}$ obtained from the input training cases. Similarly, threshold $\beta$ and $\gamma$ for the feature $F_{2}$ in the second layer are chosen to be certain values of $F_{2}$. The corresponding feature values of each input case in the training set will then be compared with the thresholds, thereby classifying the input cases into the four branches.

Step 3. In the third layer, the four thresholds $p, q, r, s$ are chosen to be four different values of feature $F_{3}$ and the input cases are further classified into eight groups. By comparing the classification obtained from the decision tree with the known true classification (gold standard), we can determine for each individual case whether it is a true positive (TP) or a false positive (FP) decision by the current tree. By summing the number of TP cases and FP cases in each branch, the total number of TP and FP cases for the chosen set of seven thresholds are obtained.

Step 4 . The algorithm determines the maximum TP value at each FP value. It does so by checking whether the current TP value is greater than the previous maximum TP value at the 
same FP value. If it is, the maximum TP value is updated and the current set of thresholds replaces the previous set for this FP value.

Step 5. Steps 3 and 4 are repeated with different combinations of $p, q, r, s$ thresholds and then steps 2-4 are repeated with all different combinations of $\alpha, \beta, \gamma$ thresholds until all possible combinations of thresholds as suggested by the input feature values of the training cases are evaluated.

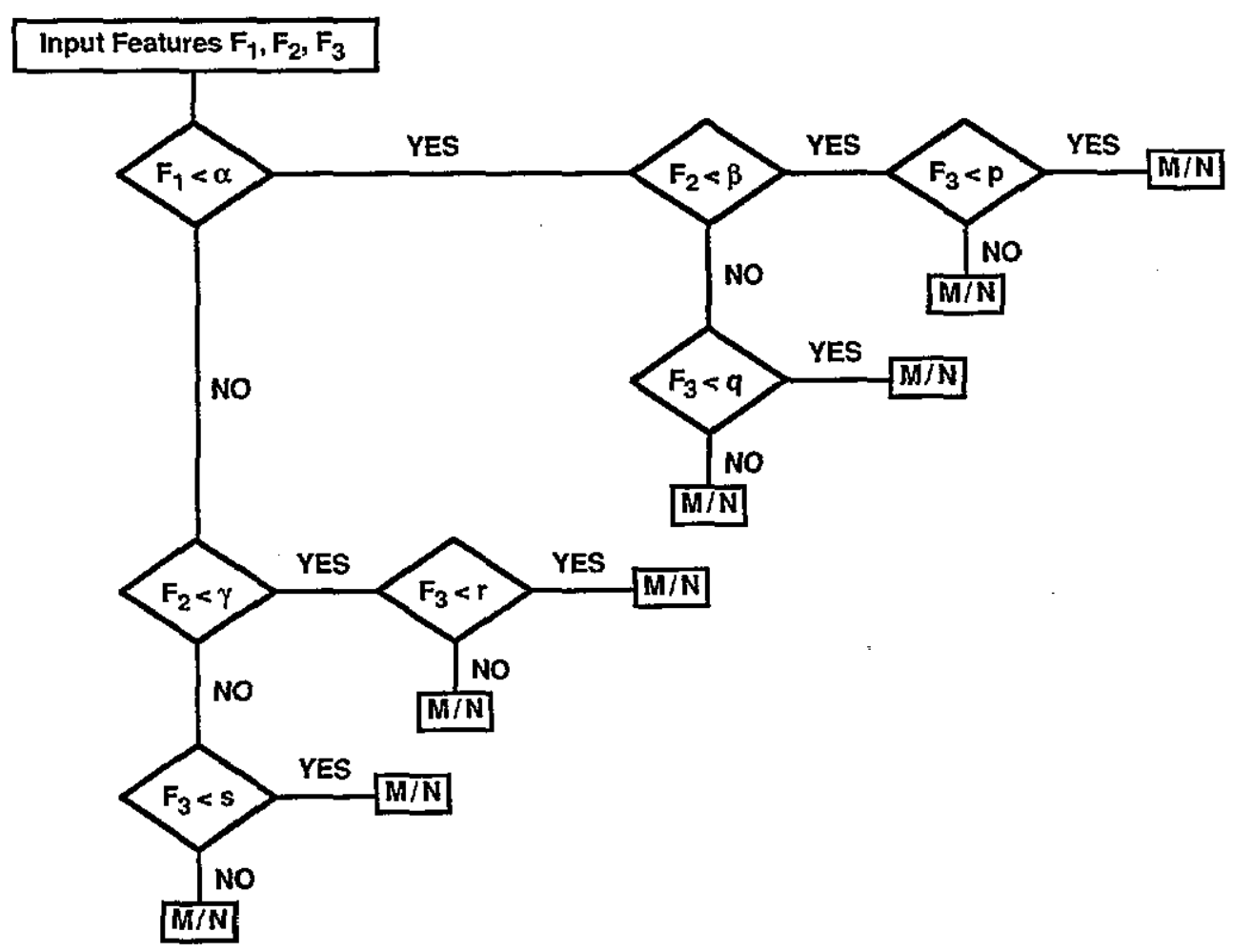

Figure 2. A flow diagram of the architecture of a three-layer, seven-node decision tree used for classification of the mass and non-mass Rors. A binary decision is made at each node using its associated decision threshold as indicated in the diagram. In the third layer, $\mathrm{M} / \mathrm{N}$ indicates that, during training, the decision tree algorithm allows the branch corresponding to either 'greater than or equal to' or 'Iess than' to be assigned to the mass class. Once the tree structure, thresholds, and direction of inequality are chosen during training, they will be fixed during test or application.

With this training procedure the highest values of TP at each possible FP value and the corresponding set of seven thresholds $\alpha, \beta, \gamma, p, q, r, s$ will be found. The classification accuracy can be evaluated on a graph plotting the true-positive fraction (TPF) against the false-positive fraction (FPF). The TPF is calculated as the ratio of mass Rols correctly identified as containing masses to the total number of mass RoIs in the input data set. The FPF is calculated as the ratio of non-mass Rols incorrectly identified as containing masses to the total number of non-mass RoIs in the input data set. Each data point on the graph represents a pair of FPF and TPF values obtained from a decision tree with its seven 
specific decision thresholds at the nodes. If may be noted that the TPF-versus-FPF curve is not a conventional receiver operating characteristic (ROC) curve that assumes that both the normal and abnormal cases follow a normal distribution and a single threshold can be applied to the decision variable. As a result, the available ROC curve-fitting programs and the associated statistical significance tests (Metz et al 1990) will not be applicable to the TPF-versus-FPF curves obtained in this study.

The classification accuracy obtained from these fixed-order trees can be compared with those from trees of different feature orders, as well as with those from trees of different feature combinations. The decision tree with the optimal feature combination and order for this task can thus be identified. Examples of the training and test results obtained in this study will be discussed below. It should be noted that, to use a trained decision-tree classifier, one has to choose the specific tree structure with the set of decision thresholds corresponding to the desired sensitivity (TPF) and specificity (1-FPF). The structure and the thresholds will be fixed during testing or application.

\section{Results}

The correlation coefficients between pairs of features within a class and the variancenormalized distances between the feature means of the mass and non-mass classes are tabulated in table 2 and table 3 , respectively. Table 2 indicates that there is a strong correlation between some of the features, for example, between energy and entropy, inertia and inverse difference moment, and inverse difference moment and difference entropy. The correlation feature is relatively independent of other features. Further, the feature distances in table 3 indicate that correlation provides the greatest separation between the mass and the non-mass Rors. These analyses thus provided a guide for selection of features for the classification task.

We first studied the effect of different feature orders for a given combination of three texture features. The results of the output FPF and TPF for distinguishing the $M$ and $N$ classes by using the features sum average, correlation, and energy are shown in figure 3 . Only three out of the six possible orders for the three features were plotted to maintain readability of the graph. The three orders shown illustrate the range of TPF obtained with different order of the features. About $10 \%$ variation in the TPF, depending on the FPF values, was possible by changing the orders of the features on the tree. The order of sum average $\left(F_{1}\right)$, correlation $\left(F_{2}\right)$, and energy $\left(F_{3}\right)$ was found to be the best for this feature combination.

The dependence of classification accuracy on feature combination was studied. Since three out of eight features were used for the tree, there were 56 possible combinations. We did not exhaustively study all 56 combinations. Rather, we considered correlation to be the most important feature based on the correlation-coefficient and distance analyses (tables 2 and 3 ) and included correlation as one of the three features in most cases. Thus, only 21 combinations were possible when two features were selected from the remaining seven features. A few combinations of three features without the correlation feature were also evaluated for comparison. The classification accuracies for four selected feature combinations, which represent the best, the worst, and two intermediate results, are shown in figure 4. For each feature combination, the results obtained with the best order were plotted. The different combinations of features could provide greater than $20 \%$ variation in TPF. The best combination appeared to be sum average-correlation-energy, whereas difference entropy-inertia-inverse difference moment was the poorest choice among those studied. These results were consistent with observation from the analysis of correlation 

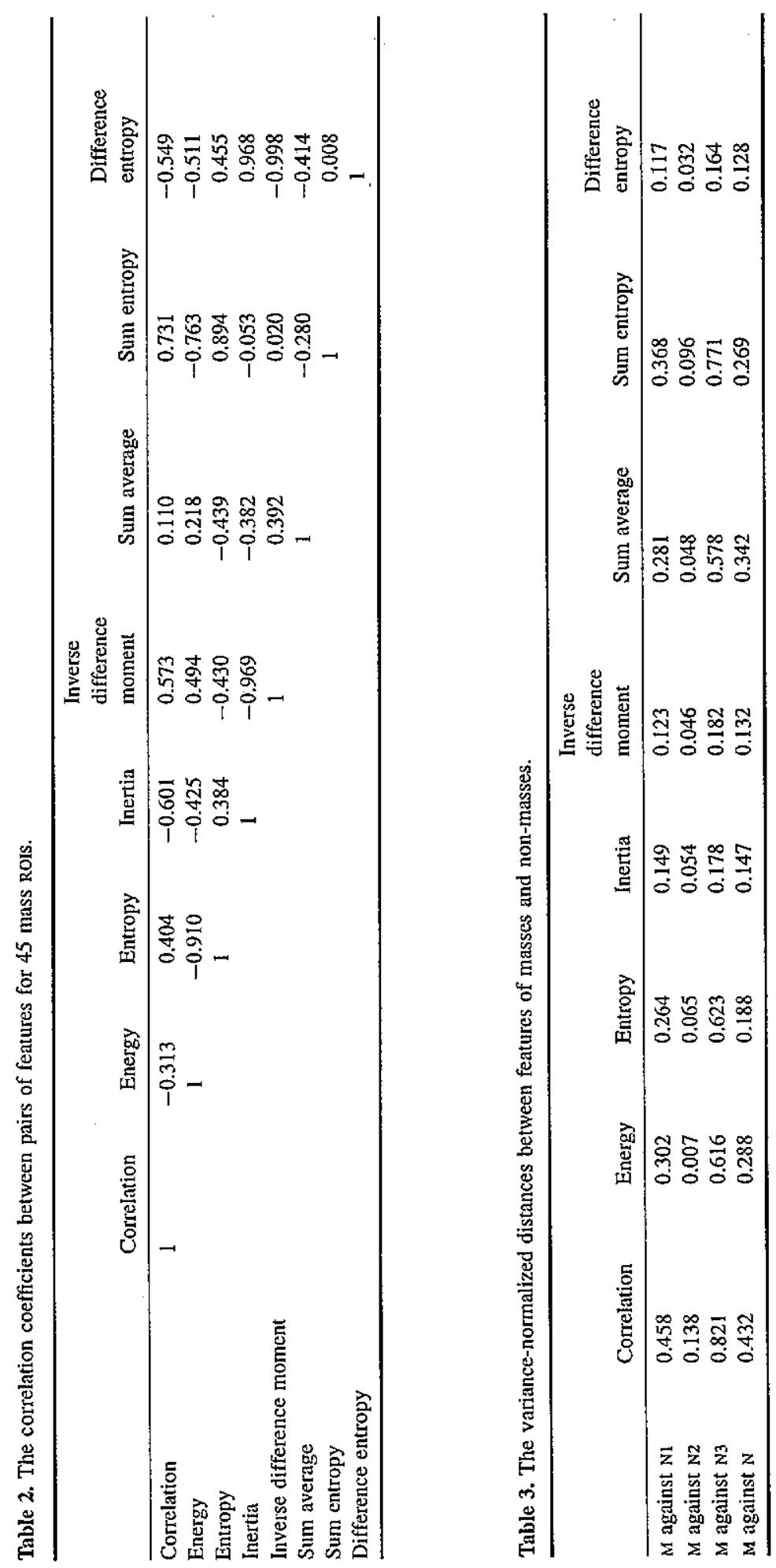


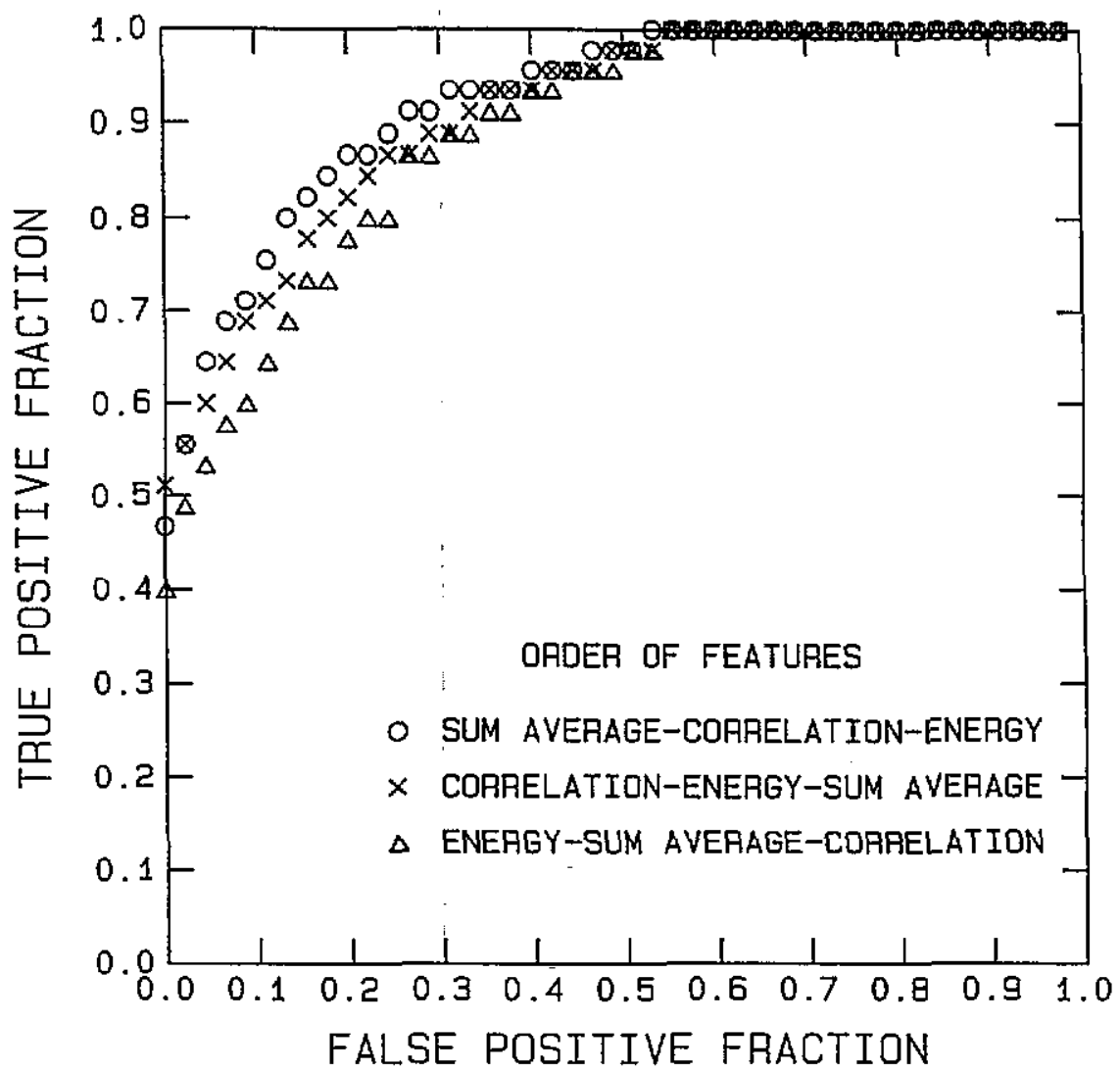

Figure 3. The effect of feature order on classification accuracy. The three features used on the tree were energy, correlation, and sum average. Three out of the six possible orders of the three features are plotted. They were chosen to illustrate the range of TPF obtained with the different orders. The best order was sum average-correlation-energy. Forty-five M-class RoIs and 45 $\mathrm{N}$-class RoIs were used for training.

coefficients and distances. Correlation, sum average, and energy were the features that had the greatest distances between the $\mathrm{M}$ - and $\mathrm{N}$-class ROIs and they were relatively independent of each other. On the other hand, difference entropy, inertia, and inverse difference moment had the shortest distances between the two classes and they were highly correlated with one another; combination of these three features on the tree might not provide much additional information in comparison with using one of the features alone, thereby resulting in poor classification.

To study the classification ability of the decision tree, using the sum average-correlationenergy features for the various $\mathrm{N}$-type ROIs, we used the $45 \mathrm{NI}, 45 \mathrm{~N} 2$, and $45 \mathrm{~N} 3$ ROIs separately in combination with the $45 \mathrm{M}$ ROIs as our input data set. Figure 5 shows the results of classification. As expected, the accuracy was highest for classification of the mass and fatty tissue (N3), intermediate for the mass and medium-dense area (N1), and lowest for the mass and dense fibroglandular tissue (N2). The classification accuracy between $M$ and N2 ROIs was about $71 \%$ TPF at an FPF of $24 \%$.

In this preliminary study, the number of case samples was small. To maximize the utility 


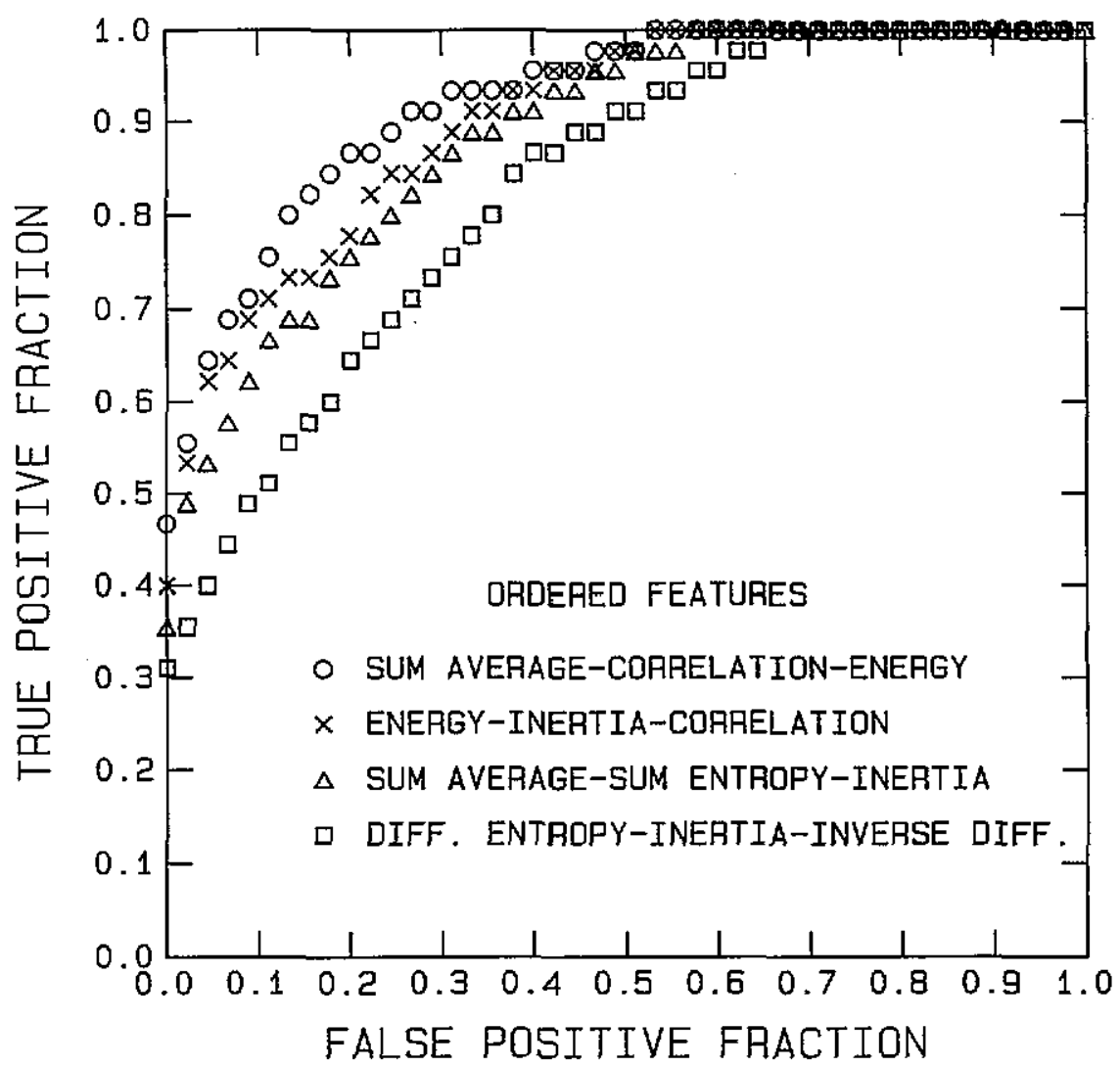

Figure 4. The effect of feature combination on classification accuracy. Out of the combinations studied, sum average-correlation-energy appeared to be the best combination. Forty-five M-class Rors and $45 \mathrm{~N}$-class Rors were used for training.

of the available cases for training, we used a leave-one-out method (Jain 1989) for testing the decision tree classifier (sum average-correlation-energy). In each training cycle, we left out both the $\mathrm{M}$ - and the $\mathrm{N}$-class RoIs that were extracted from the same mammogram as test ROIs; the remaining $44 \mathrm{M}$ and $44 \mathrm{~N}$ Rols were used for training the tree. This eliminated the possibility that the trained tree learned about the structured background of a specific mammogram from the ROI extracted from the same mammogram, as might be in the case when one ROI was included for training and the other from the same mammogram for testing. The results of the leave-one-out test are shown in figure 6 . Because there are actually 45 training curves, one for each training cycle, the plotted training curve is the one obtained with the complete set of $45 \mathrm{M}$ and $45 \mathrm{~N}$ Rols as training set, i.e., the same curve as that shown in figure 4 . We had verified that the training curves in the leave-one-out test were almost indistinguishable from the training curve with the complete data set. Each of the data points on the training curve has a corresponding test point. The number of test points appears to be fewer because some of them overlap. A pair of the training and test results is marked with solid symbols as an example. This tree yielded a TPF of about $89 \%$ and an FPF of $24 \%$ in each training cycle. For this trained tree with the set of seven thresholds, the test result was about $76 \%$ TPF at an FPF of $36 \%$. It should be noted that 


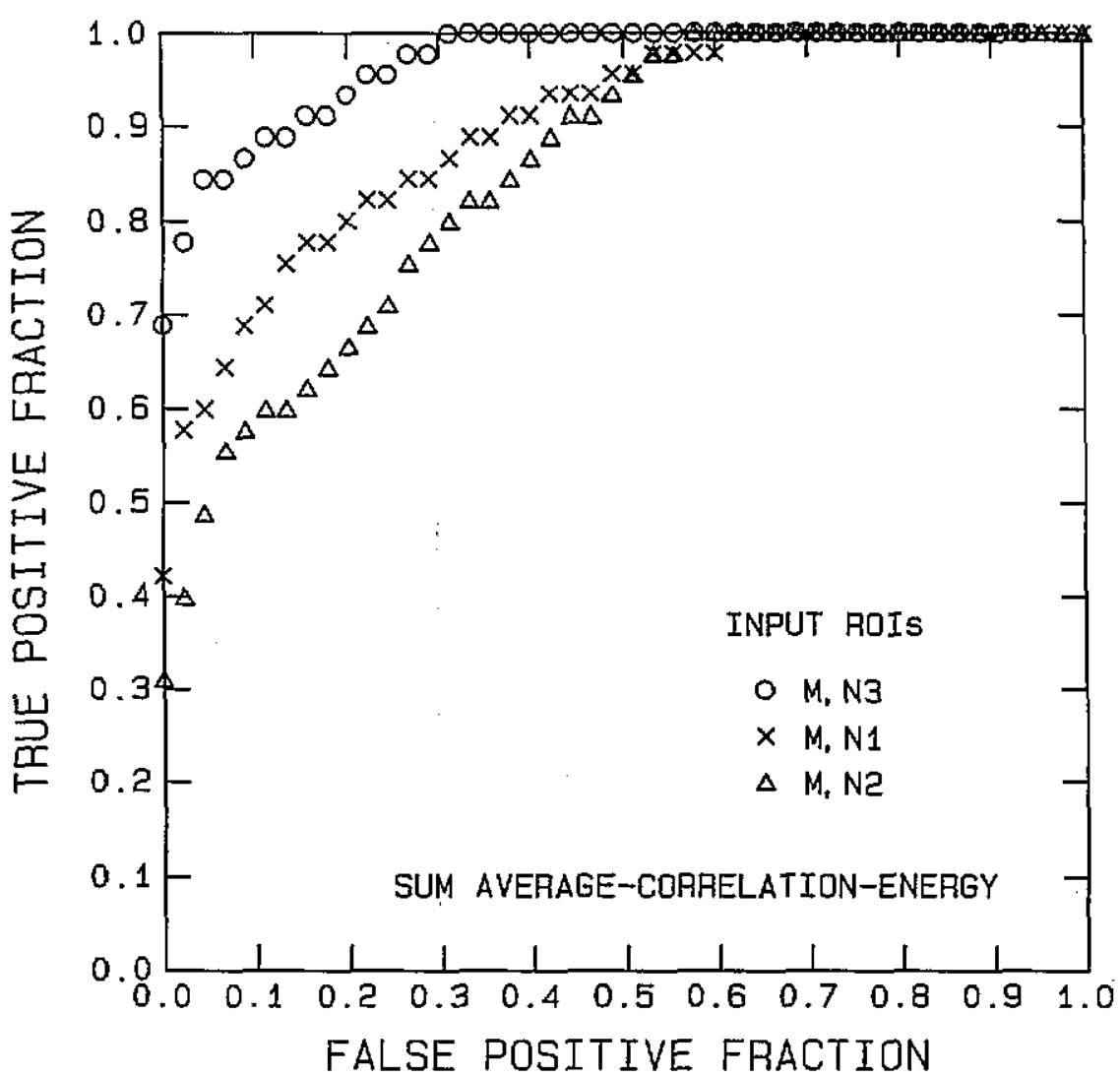

Figure 5. The accuracy of the decision tree (sum average-correlation-energy) for ciassification of the mass Rors and the three types of normal RoI. Forty-five M Rors and $45 \mathrm{~N}$-type Rors were used in each case.

any of the trained trees indicated by the circular data points can be chosen as an operating point, as in the ROC analysis. However, there is a trade-off between the TPF and the FPF. A high sensitivity is always accompanied by a low specificity. The selection of an 'optimal' operating point has to be based on the specific task for which this texture-analysis method will be used and cost-benefit considerations for the task.

\section{Discussion}

In this work we investigated whether texture features of mammographic breast patterns could provide reliable distinction between mass and non-mass regions. The classification accuracy, represented by the curves shown in figures $3-6$, while encouraging, is inadequate for practical clinical implementation. However, the method proposed here provides the flexibility to determine the specifically ordered sequences of various texture features (or tree branches) in order to achieve desirable separation of classes. In other words, an advantage of the decision-tree classification technique employed is that it is robust. Its performance can be optimized for a specific task by combining features and comparing results for different arrangements of the nodes for the various texture features. 


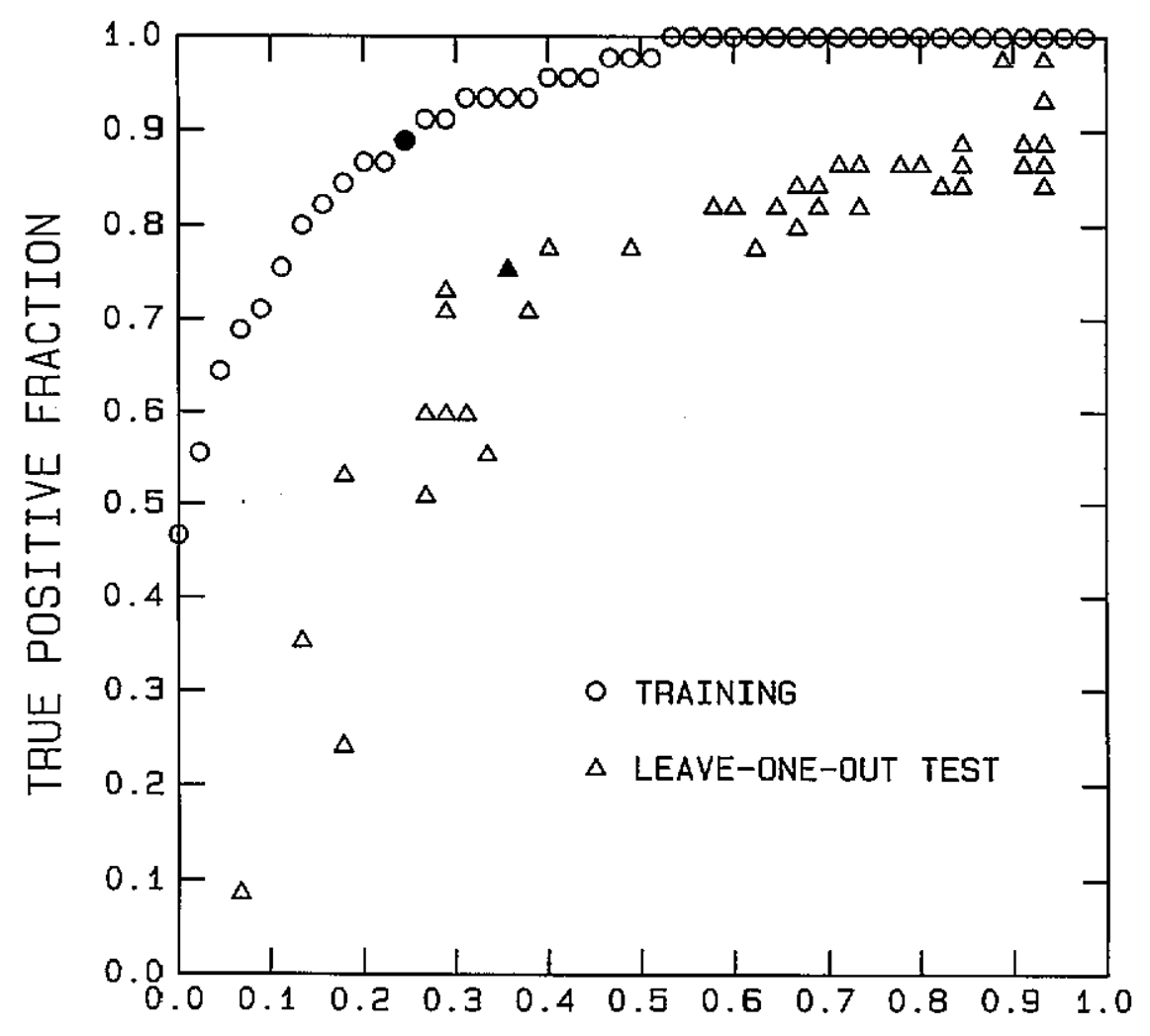

FALSE POSITIVE FRACTION

Figure 6. A comparison of training and test results obtained with a leave-one-out method. Results for 45 decision-tree classifiers, each corresponding to one point on the training curve, were shown. The test result indicated by a solid triangle was obtained with the decision tree indicated by a solid square on the training curve.

In our current algorithm described above, the optimal decision threshold in each of the tree nodes is obtained by exhaustively searching through all combinations of threshold values to achieve the maximum TPF. The CPU time required for this procedure increases rapidly with increasing number of input training cases. An alternative approach that yields near-optimal threshold values may be employed. In this alternative search procedure, once the distributions of the $F_{3}$ feature values in the four nodes are obtained, the sensitivity of the TP values for a desired FPF in each of the nodes may be maximized separately. For example, suppose that in the $i$ th node a number of $\mathrm{M}$ - and $\mathrm{N}$-feature values are obtained. By comparing the average values of the $M$ and $N$ features, the algorithm can determine whether the branch corresponding to 'greater than or equal to' or 'less than' should be assigned to the mass class. The threshold is then set only by the values of either the $M$ or $\mathrm{N}$ features, and the maximum possible values of TP at a predetermined FPF (e.g. 20\%) are calculated for each node. We observed that, for the limited preliminary comparisons that we made, the TPF obtained with this approach was a few per cent lower than that obtained with the exhaustive search procedure. Alternatively, in the case of a large training data set such that a distribution of feature values can be formed at each node, one can perform 
thresholding of the feature space in a systematic way by using uniform division of the feature values for the distribution at each node for decision making. This third method can be advantageous over the current method in terms of computation time and over the second method in terms of precision. This further demonstrates the flexibility of using different optimization procedures in the tree construction process.

In this preliminary study, we constructed SGLD matrices in four directions $\left(\theta=0^{\circ}, 45^{\circ}\right.$, $90^{\circ}, 135^{\circ}$ ) at a pixel distance of $d=1$. We observed no substantial difference in the feature properties calculated in the four directions. Therefore, an average of the feature values over the four directions were used as input to the classifier to simplify feature selection. Furthermore, to reduce the computation time and to improve the statistics of the SGLD matrix, the grey-level depth of the ROI images had to be reduced from 12 bits. Because the three least significant bits of the 12-bit images contained mainly screen/film noise (Chan et al 1994) and we found that there was no substantial difference in the classification accuracy when the pixel depth was varied from nine bits to seven bits, the SGLD matrices were calculated at seven bits. Note that for the detection of microcalcifications, it was found that the detection accuracy decreased significantly if the grey-level resolution was reduced to less than nine bits (Chan et al 1994). The detection of microcalcifications is a fundamentally different task from the texture analysis of masses and normal breast tissue. It can be expected that both the spatial and grey-level resolution requirements for the former task are much more stringent than those for the latter because of the subtle nature of the microcalcifications. More detailed analyses of the dependence of the classification accuracy for mass and normal tissue on the distance and bit depth in construction of the SGLD matrices, as well as on the directionality of the texture features, will be performed in future studies.

Many texture features derived from different methods (Haralick 1986) may be useful for differentiation of masses and normal tissue. In this feasibility study, we have not attempted to investigate the performance of different texture features. We chose the texture features calculated from the SGLD matrix because it was reported that they were effective in distinguishing different types of texture (Haralick et al 1973). Since normal tissue that mimics a mass is usually a result of overlapping mammographic structures, it is expected that normal tissue should have more complex textures than solid masses. The results of our study indicated that texture features from the SGLD matrix contained information for distinguishing normal tissue from masses. Further investigation to compare the effectiveness of various texture features in this application is warranted.

The goal of this study was to investigate whether texture features based on the SGLD matrix can be used for distinguishing mass and normal tissue. Such methods can be implemented in a computer-aided diagnosis program, which automatically screens an input mammogram for suspicious areas, to reduce false detections. Alternatively, such methods can be implemented in an interactive display station, which may be developed in the future for digital mammography, to provide a second opinion on the probability that a suspicious area identified by a radiologist may contain a mass.

The large difference in the training and test results suggests that the training data set in this study is not large enough to provide optimal decision thresholds for the test cases. This may be improved by increasing the number of training cases in future development. A larger number of texture or morphological features may also be used to improve the classification accuracy either between mass and normal tissue or to classify between benign and malignant masses. Image preprocessing in terms of background reduction, noise suppression, or signal enhancement will need to be explored. Optimization of the feature characteristics by studying their dependence on the parameters in the SGLD matrix will need to be performed. The efficiency and accuracy of the decision-tree classifier in comparison with other classifiers 
will also need to be investigated. The present study concentrated on evaluation of the feasibility of using texture features derived from the SGLD matrix for classification of mass and normal tissue. Our results indicate that the texture features will probably be useful for classifying true and false detections in computer-aided diagnosis programmes.

\section{Acknowledgments}

This work is supported by USPHS grant CA 48129, a Faculty Research Award (FRA334) from the American Cancer Society and US Army grant DAMD 17-93-J-3007 (through subgrant GU RX 4300-803UM from Georgetown University). The content of this publication does not necessarily reflect the position of the university or the government and no official endorsement of any equipment or product of any companies mentioned in the publication should be inferred. The authors are grateful to Diane Williams for secretarial assistance.

\section{Appendix A. The spatial-grey-level-dependence (SGLD) matrix and texture features}

The $(i, j)$ th element of the SGLD matrix, $p_{\theta, d}(i, j)$, is the joint probability that the grey levels $i, j$ occur in a direction of angle $\theta$ and at a distance of $d$ pixels apart over the entire ROI. The joint probability $p_{\theta, d}(i, j)$ is normalized by the number of grey-level pairs obtained from the ROI with a pixel distance of $d$. For each ROI eight features were derived from its SGLD matrix as described below. A simplified notation $p(i, j)$ will be used for the SGLD matrix elements in the formulation

$$
\text { Energy }=\sum_{i=0}^{n-1} \sum_{j=0}^{n-1}[p(i, j)]^{2}
$$

where $n$ is the number of grey levels per pixel.

$$
\text { Correlation }=\left(\sum_{i=0}^{n-1} \sum_{j=0}^{n-1}\left(i-\mu_{x}\right)\left(j-\mu_{y}\right) p(i, j)\right) / \sigma_{x} \sigma_{y}
$$

where

$$
\begin{aligned}
\mu_{x} & =\sum_{i=0}^{n-1} i \sum_{j=0}^{n-1} p(i, j) & \sigma_{x}^{2} & =\sum_{i=0}^{n-1}\left(i-\mu_{x}\right)^{2} \sum_{j=0}^{n-1} p(i, j) \\
\mu_{y} & =\sum_{j=0}^{n-1} j \sum_{i=0}^{n-1} p(i, j) & \sigma_{y}^{2} & =\sum_{j=0}^{n-1}\left(j-\mu_{y}\right)^{2} \sum_{i=0}^{n-1} p(i, j)
\end{aligned}
$$

are the mean and variance of the marginal distributions $p_{x}(i)$ and $p_{y}(j)$, respectively.

$$
\text { Inertia }=\sum_{i=0}^{n-1} \sum_{j=0}^{n-1}(i-j)^{2} p(i, j)
$$




$$
\begin{aligned}
& \text { Entropy }=-\sum_{i=0}^{n-1} \sum_{i=0}^{n-1} p(i, j) \log _{2} p(i, j) \\
& \text { Inverse difference moment }=\sum_{i=0}^{n-1} \sum_{j=0}^{n-1} \frac{1}{1+(i-j)^{2}} p(i, j) \\
& \text { Surn average }=\sum_{k=0}^{2 n-2} k p_{x+y}(k)
\end{aligned}
$$

where

$$
\begin{aligned}
& p_{x+y}(k)=\sum_{i=0}^{n-1} \sum_{j=0}^{n-1} p(i, j) \quad i+j=k \quad k=0, \ldots, 2 n-2 \\
& \text { Sum entropy }=-\sum_{k=0}^{2 n-2} p_{x+y}(k) \log _{2} p_{x+y}(k) \\
& \text { Difference entropy }=-\sum_{k=0}^{n-1} p_{x-y}(k) \log _{2} p_{x-y}(k)
\end{aligned}
$$

where

$$
p_{x-y}(k)=\sum_{i=0}^{n-1} \sum_{j=0}^{n-1} p(i, j) \quad|i-j|=k \quad k=0, \ldots, n-1
$$

These texture features describe the shape of the SGLD matrix, which is related to specific textural characteristics of the image such as homogeneity, contrast, and the presence of organized structures within the ROI. They reflect the complexity and the nature of grey-tone transitions that occur in the image. However, it is difficult to identify which specific textural characteristic is represented by each of these features (Haralick et al 1973, Kruger et al 1974).

\section{Appendix B. The correlation coefficient and variance-normalized distance of features} (Stark and Woods 1986)

To evaluate the correlation coefficient between any two of the features $F_{1}$ and $F_{2}$ within a class (table 2), we made use of the following relationship:

Correlation coefficient $\left(F_{1}, F_{2}\right)$

$$
=\left(\frac{1}{m} \sum_{i=1}^{m}\left[f_{1, i}-\operatorname{ave}\left(F_{1}\right)\right]\left[\left(f_{2, i}-\operatorname{ave}\left(F_{2}\right)\right]\right) / \sqrt{\operatorname{var}\left(F_{1}\right) \operatorname{var}\left(F_{2}\right)}\right.
$$

where $m$ is the number of $F_{1}$ or $F_{2}$ feature values, $f_{1 . i}$ and $f_{2, i}$ are the individual feature values of $F_{1}$ and $F_{2}$, respectively, calculated for the $i$ th image, and ave $\left(F_{1}\right)$, ave $\left(F_{2}\right)$, $\operatorname{var}\left(F_{1}\right), \operatorname{var}\left(F_{2}\right)$ are the averages and variances of the features, respectively. 
For estimation of the ability of a feature $F$ to distinguish between two classes, mass and non-mass, the variance-normalized distance (table 3 ) is used:

$\operatorname{Distance}\left(F_{\text {mass }}, F_{\text {non-mass }}\right)=\left|\operatorname{ave}\left(F_{\text {mass }}\right)-\operatorname{ave}\left(F_{\text {non-mass }}\right)\right| / \sqrt{\operatorname{var}\left(F_{\text {mass }}\right)+\operatorname{var}\left(F_{\text {non-mass }}\right)}$

where ave $\left(F_{\text {mass }}\right), \operatorname{var}\left(F_{\text {mass }}\right)$ and ave $\left(F_{\text {non-mass }}\right), \operatorname{var}\left(F_{\text {non-mass }}\right)$ are the averages and variances of the feature $F$ calculated for the mass and non-mass Rors, respectively.

\section{References}

Agin G J and Duda R O 1975 SRI vision research for advanced automation Proc. 2nd USA-Japan Computer Conf. (Tokyo) pp 113-7

Brzakovic D, Luo X M and Brzakovic P 1990 An approach to automated detection of tumors in mammograms IEEE Trans. Med. Imaging MI-9 233-4I

Caldwell CB, Stapleton S J, Holdsworth D W, Jong R A, Weiser W J, Cooke G and Yaffe M J 1990 Characterization of mammographic parenchymal pattern by fractal dimension Phys. Med. Biol. 35 235-47

Chan H P, Doi K, Galhotra S, Vyborny C J, MacMahon H and Jokich P M 1987 Image feature analysis and computer-aided diagnosis in digital radiography. L. Automated detection of microcalcifications in mammography Med. Phys. $14538-48$

Chan H P, Doi K, Vyborny C J, Schmidt R A, Metz C E, Lam K L, Ogura T, Wu Y and MacMahon H 1989 Improvement of radiologists' detection of clustered microcalcifications on mammograms: the potential of computer-aided diagnosis Invest. Radiol. $251102-10$

Chan H P, Niklason L T, Ikeda D M, Lam K L and Adler D D 1994 Digitization requirements in mammography: effects on computer-aided detection of microcalcifications Med. Phys. 21 1203-11

Cheng S N C, Chan H P, Helvie M A, Goodsitt M M, Adler D D and StClair D 1993 Classification of mass and non-mass regions on mammograms using artificial neural network J. Imaging Sci. Technol. at press

Cheng S N C, Chan H P, Niklason L T, Adler R S and Chang C L 199I Texture analysis of trabecular patterns on phalanges using neural network Radiology P 181143

Conners R W 1979 Towards a set of statistical features which measure visually perceivable qualities of textures Proc. IEEE Conf. on Pattern Recognition and Image Processing pp 382-90

Davies D H and Dance D 1990 Automatic computer detection of clustered calcifications in digital mammograms Phys. Med. Biol. $351111-8$

Dhawan A P, Chitre Y S and Moskowitz M 1993 Artificial-neural-network-based classification of mammographic microcalcifications using image structure features Proc. SPIE 1905 820-31

Fam B W, Olson S L, Winter P F and Scholz F J 1988 Algorithm for the detection of fine clustered calcifications on film mammograms Rudiology $169333-7$

Garra B S, Insana M F, Shawker T U, Wagner R F, Bradford M and Russell M A 1989 Quantitative ultrasonic detection and classification of diffuse liver disease. Comparison with human observer performance Invest. Radiol. 24 196-203

Goldberg V, Manduca A, Ewert D L, Gisvold J J and Greenleaf J F 1992 Improvement in specificity of ultrasonography for diagnosis of breast tumors by artificial intelligence Med. Phys. 19 1475-81

Hand W, Semmlow J L, Ackerman L V and Alcorn F S 1979 Computer screening of xeromammograms: a technique for defining suspicious areas of the breast Comput. Biomed. Res. $12445-60$

Haralick R M 1986 Statistical image texture analysis Handbook of Pattern Recognition and Image Processing (New York: Academic) ch 11

Haralick R M, Shanmugam K and Dinstein I 1973 Texture features for image classification IEEE Truns. Syst. Man Cybernet. SMC-3 610-21

Jain A 1989 Fundamentals of Digital Image Processing (Englewood Cliffs, NJ: Prentice-Hall)

Katsuragawa S, Doi K and MacMahon H 1988 Image feature analysis and computer-aided diagnosis in digitial radiography: detection and characterization of interstitial lung disease in digital chest radiographs Med. Phys. $15311-9$

Kegelmeyer W P, Pruneda J M, Bourland P D, Hillis A, Riggs M W and Nipper M L 1994 Computer-aided mammographic screening for spiculated lesions Radinlogy 191 331-7

Kruger R P, Thompson W B and Turner A F 1974 Computer diagnosis of pneumoconiosis IEEE Trans. Syst. Man Cybernet. SMC-4 40-9 
Lai S M, Li X and Bischof W F 1989 On techniques for detecting circumscribed masses in mammograms IEEE Trans. Med. Imaging MI-8 377-86

Magnin I E, Cluzeau F, Odet CL and Bremond A 1986 Mammographic texture analysis: an evaluation of risk for developing breast cancer Opt. Eng. $25780-4$

Mascio L N, Hernandez J M and Logan C M 1993 Automated analysis for microcalcifications in high-resolution digital mammograms Proc. SPIE 1989 472-9

Metz C E, Shen J H and Herman B A 1990 New methods for estimating a binormal koc curve from continuouslydistributed test results 1990 Annual Meeting Am. Stot. Assoc. (Anaheim, CA, 1990)

Pratt W K 1978 Digital Image Processing (New York: Wiley)

Stark H and Woods J W 1986 Probability, Random Processes and Estimation Theory for Engineers (Englewood Cliffs, NJ: Prentice-Hall)

Yin F F, Giger M L, Doi K, Metz C E, Vyborny C J and Schmidi R A 1991 Computerized detection of masses in digital mammograms: analysis of bilateral subtraction images Med. Phys. 18 955-63 